\title{
Single arm approach for transcatheter aortic valve implantation
}

\author{
Jerzy Sacha1,2, Krzysztof Krawczyk¹, Witold Gwóźdź³ , Radosław Gawroński \\ ${ }^{1}$ Department of Cardiology, University Hospital, Institute of Medical Sciences, University of Opole, Opole, Poland \\ ${ }^{2}$ Faculty of Physical Education and Physiotherapy, Opole University of Technology, Opole, Poland \\ ${ }^{3}$ Department of Cardiac Surgery, University Hospital, Institute of Medical Sciences, University of Opole, Opole, Poland
}

Adv Interv Cardiol 2021; 17, 3 (65): 322-323

DOI: https://doi.org/10.5114/aic.2021.109148

The fully percutaneous femoral approach for transcatheter aortic valve implantation (TAVI) is now the gold standard for enabling early patient ambulation. However, an alternative route through the subclavian-axillary access may offer some advantages for rapid mobilization. We present the TAVI technique performed only with the single-arm approach, i.e. in a way that brings us closer to the standards of coronary procedures through radial access.

An 86-year-old man was qualified for TAVI via a subclavian-axillary access due to the tortuosity of calcified iliac arteries and the frailty syndrome. The procedure was performed under conscious sedation. First, a 7F sheath and a long safety guidewire were inserted through the left radial access, and then angiography was performed to identify the puncture site (Figure $1 \mathrm{~A}$ ). Under ultrasound guidance, the left axillary artery was punctured two centimeters below the clavicle and three ProGlide sutures and a $14 \mathrm{~F}$ sheath were placed. Using a safety guidewire, a $6 \mathrm{~F}$ pigtail catheter was inserted into the aortic root for contrast injections during TAVI. The aortic valve was crossed with a regular straight guidewire that was replaced with the Confida stiff guidewire, which was also used as an electrode for rapid pacing. Valvuloplasty was performed with a $23 \mathrm{~mm}$ balloon and then the Evolut $\mathrm{R} 34 \mathrm{~mm}$ valve was implanted (Figure $1 \mathrm{~B}$ ). It is noteworthy that the minimum diameter of the subclavian artery determined on angio-CT was $8 \mathrm{~mm}$ and housed both the Evolut $\mathrm{R}$ $34 \mathrm{~mm}$ delivery system and the $6 \mathrm{~F}$ pigtail catheter. After the implantation, the safety guidewire was inserted through the pigtail catheter, which was subsequently replaced with the peripheral $10 \mathrm{~mm}$ balloon. The balloon diameter was selected in the ratio $1: 1$ to the diameter of the axillary artery at the puncture site determined in the ultrasound examination. While removing the delivery system, the peripheral balloon was inflated to prevent bleeding from the axillary access site (Figure $1 \mathrm{C}$ ), and then the puncture site was closed with ProGlide and Angio-Seal 8F. The contrast injection through the radial sheath confirmed a good hemostatic effect. Almost immediately after the procedure, the patient was able to walk and his hand looked like after a coronary intervention via a radial access (Figure $1 \mathrm{D}$ ).

The presented single arm approach is a good alternative way for TAVI not only in the absence of femoral access, but also in patients where early mobilization is important, e.g. elderly frail subjects or those at a high risk of deep vein thrombosis. The biggest problem with this technique is the risk of bleeding from the subclavian-axillary access site, which is potentially difficult to compress. However, the safety guidewire (inserted through the radial artery) permits the insertion of a peripheral balloon and tamponade of the bleeding site [1]. For more serious vascular complications, such as vessel dissection or major bleeding, the safety guidewire enables the delivery of self-expanding stents or stent grafts [2]. Importantly, for the effective percutaneous closure of the access site, it is crucial to puncture the artery in a place free from calcified plaques, so it should always be done under ultrasound guidance [1-3].

\section{Conflict of interest}

The authors declare no conflict of interest.

\section{Corresponding author:}

Jerzy Sacha MD, PhD, Department of Cardiology, University Hospital, Institute of Medical Sciences, University of Opole, Opole, Poland, phone: +487745208 72, e-mail: sacha@op.pl

Received: 5.04.2021, accepted: 15.06.2021. 


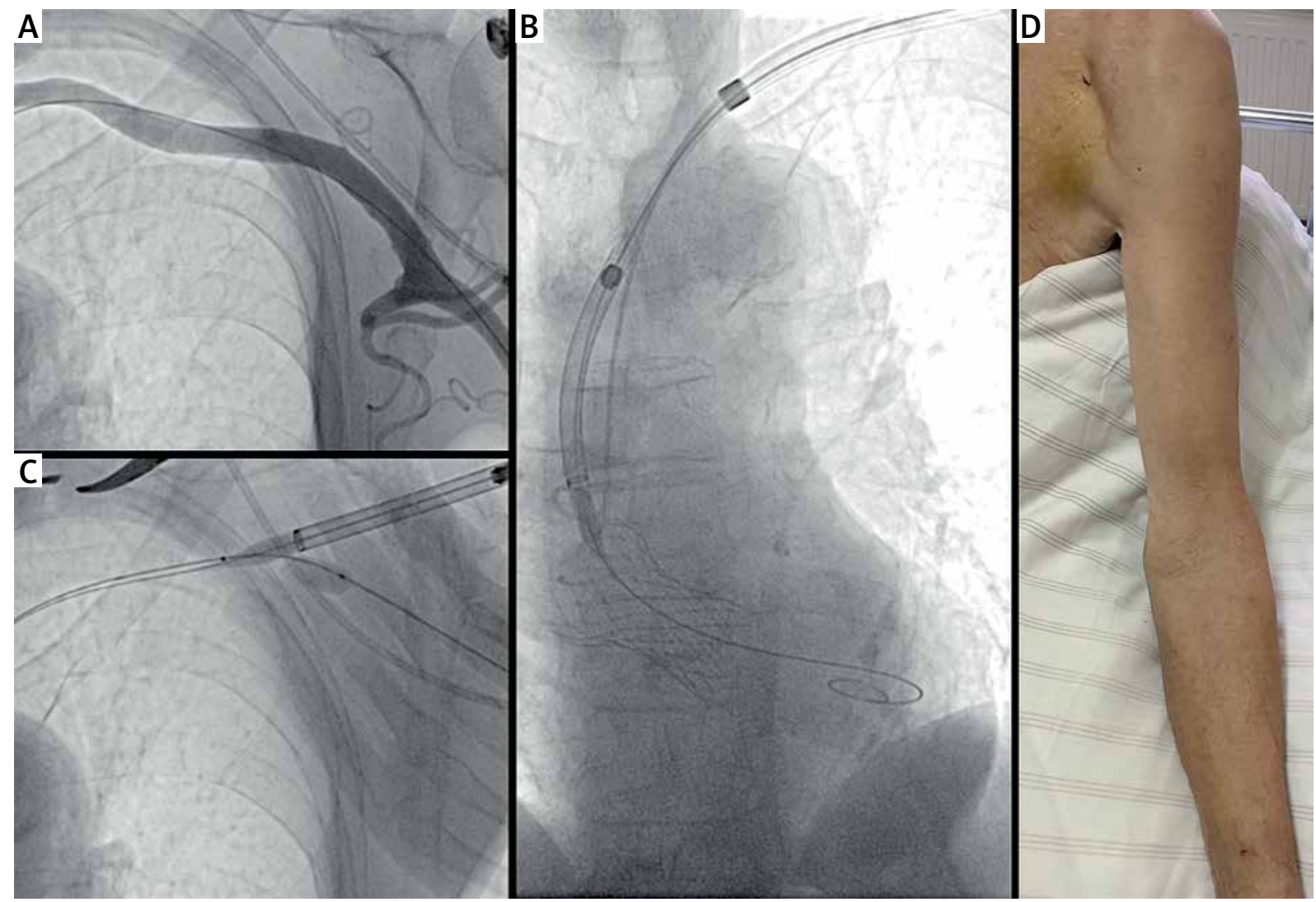

Figure 1. Single arm approach for TAVI. A - Retrograde contrast injection through the radial sheath (the safety guidewire was inserted via the radial access to the ascending aorta). B - Full TAVI through the left arm, i.e. the pigtail catheter was inserted via the left radial access, but the valve was implanted through the left axillary access (the left ventricle was paced with the Confida guidewire). C - Balloon tamponade of the access site to prevent bleeding during removal of the delivery system. D - Axillary and radial access sites after the procedure (i.e. after removing the radial compression band)

\section{References}

1. Sacha J, Krawczyk K, Gierlotka M. Fully percutaneous insertion and removal of the Impella CP via a subclavian approach. Adv Interv Cardiol 2020; 16: 343-6.

2. Sacha J, Krawczyk K, Gwóźdź W, et al. Fully percutaneous transaxillary aortic valve replacement with effective bailout plan for vascular complications. JACC Cardiovasc Interv 2020; 13: 2811-2.

3. Sacha J, Krawczyk K, Gawor M, et al. Balloon aortic valvulopasty, Impella insertion and complex coronary intervention: is this all feasible fully percutaneously via upper limbs access. Adv Interv Cardiol 2021; 17: 126-8. 